\title{
Three dimensional reconstruction of the heart with rotational acquisition: methods and clinical applications
}

\author{
Alessandro Salustri, Jos Roelandt
}

\begin{abstract}
Advances in ultrasound instrumentation have introduced a new era in cardiovascular imaging. The multiplane transoesophageal probe represents the most recent technological innovation and allows improved and more effective imaging of the heart in multiple sequential planes by circumventing chest wall problems. ${ }^{1}$ However, as with all other approaches, images are obtained in a two dimensional format. Thus cardiologists have the difficult task of forming a mental concept of the three dimensional relationships of complex cardiac structures. This process is not always straightforward or accurate, especially in the presence of complex pathology. Consequently, the acquisition and display of images of the heart in three dimensional space has been attempted for more than a decade, but several technical limitations have hampered its clinical acceptance. ${ }^{2-5}$ In particular, the quality of precordial images has often been less than optimal and the time required for reconstruction of the images prohibitively long. The improvement in echocardiographic equipment and the advances in computer technology have partially overcome these problems, leading to increased interest in this method. ${ }^{6}$ Moreover, the oesophageal approach offers a relatively stable site for the imaging probe and, together with the superior quality of the tomographic images, represents an ideal gateway to three dimensional reconstruction.?
\end{abstract}

At the Thoraxcenter, we have developed a prototype instrument for rotational acquisition of both transthoracic and transoesophageal echocardiographic images during a routine study. Our initial experience with this technique is promising and confirms that three dimensional echocardiography is approaching clinical application. -10 $^{-10}$

In this paper we present the basic steps required for three dimensional reconstruction of cardiac images with this technique (table 1), review its current use, and describe its potential clinical value.

\footnotetext{
Academic Hospital Rotterdam-Dijkzigt and Erasmus University, Rotterdam, The Netherlands A Salustri J Roelandt Correspondence to: Dr A Salustri, Ospedale Sandro Pertini, Via Mont Tiburtini, 00153 Rome, Italy.
}

Table 1 Requirements for three dimensional reconstruction with a rotatable transducer

(1) ACQUISITION (sequential rotational cardiac cross
sections with spatial and temporal information)
(2) PROCESSING (resampling and conversion from polar to
Cartesian coordinates)
(3) INTERPOLATION (filling the space between individual
cross sections)
(4) ENHANCEMENT (noise suppression)
(5) DISPLAY (anyplane echocardiography; volume rendered
images)

\section{The image acquisition technique}

The basic cross sectional images are sequentially collected following predetermined rotational movement of the transducer. However, for three dimensional reconstruction accurate spatial and temporal information on the basic cross sectional images is required. Indeed, the position and orientation of the heart relative to the imaging plane vary during the cardiac and respiratory cycles, and the tomographic image must be timed in the heart cycle. Spatial registration is controlled by a computer algorithm logic which controls the image acquisition in a given plane at a predetermined moment in the respiratory cycle. Temporal information is obtained by measuring heart cycle variation by ECG gating.

\section{ROTATIONAL SCANNING}

The imaging plane is rotated around its central axis by a computer controlled system (propeller-like). Usually, $2^{\circ}$ steps are used to obtain a dataset with a conical volume (fig 1 ).

Rotational scanning is ideal for three dimensional reconstruction and offers some advantages over other scanning modes. ${ }^{11}$ In particular, the small acoustic window which is required allows the operator to focus on areas of specific diagnostic importance throughout the acquisition time, resulting in optimal data sampling and thus better final image quality.

However, with the rotational technique it is extremely important that the first and the last images acquired are mirror images to avoid a mismatch between images in the conical volume, which will result in artefacts in the final display. Thus the transducer position and its central axis of rotation must remain fixed during the full $180^{\circ}$ arc sampling.

\section{THE OESOPHAGEAL PROBE}

Any of the available multiplane transoesophageal probes can be used provided the rotation of the transducer array can be controlled to obtain predetermined sequential images with spatial and temporal registration. This can be realised by a computer controlled step motor externally interfaced to the control knob of the transducer array. We used a multiplane probe (Delft Instruments Medical Imaging BV, Delft, The Netherlands) housing a $5 \mathrm{MHz}, 64$ element rotational array transducer. After being positioned in the oesophagus, the probe can be kept in a steady position by locking the antero-posterior flexion control in the anterior position. The scanning plane can be continuously rotated through $180^{\circ}$, starting from a longitudinal imaging position, 


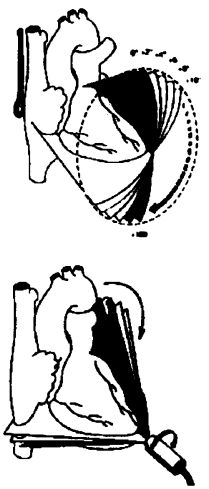

A

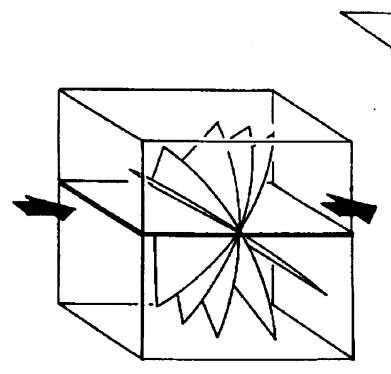

B

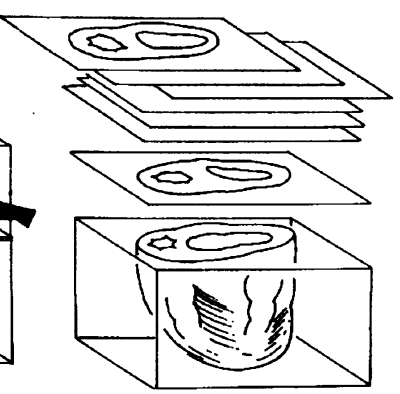

C
Figure 1 Schematic drawing showing the steps of acquisition, resampling, and conversion of echocardiographic images acquired with rotational technique. Basic cross sectional images can be acquired from either a transoesophageal or a precordial window (panel $A$ ). The digitised images are then resampled in the correct spatial sequence (panel B). Finally, conversion from polar to Cartesian coordinates is necessary for identification of each individual point in a rectangular coordinate system and further three dimensional reconstruction. by a control knob on the handle of the echoscope. The control knob of the multiplane probe is mechanically rotated by a step motor through a custom built wheel-work interface which permits step progress of the imaging plane over a span of $180^{\circ}$ to produce a conical data volume with its apex in the transducer.

\section{THE PRECORDIAL PROBE}

A commercially available transducer is mounted in a prototype housing with a cogwheel which fits into a cylindrical holder. The step motor is mounted on the holder and, through a wheel-work interface, rotates the cogwheel and the transducer inside the holder. A microswitch controls the start at $0^{\circ}$ and the end at $180^{\circ}$ of the image acquisition.

THE ULTRASOUND EQUIPMENT AND

RECONSTRUCTION SYSTEM

The probes are connected to a commercially available instrument and the video output is interfaced with the three dimensional reconstruction system (Echo-scan system, Tom Tec, Munich, Germany). This system consists of a steering system for the computer controlled image acquisition and the software for three dimensional reconstruction and display.

\section{THE IMAGE ACQUISITION}

The step motor controlling transducer rotation (either oesophageally or precordially) is activated by the steering logic which allows optimal temporal and spatial registration of a cardiac cross section by considering heart cycle variation by ECG gating and respiratory cycle variation by impedance measurement. When a cardiac cycle is selected by the steering logic and corresponds to a preselected respiratory phase, the cardiac cross section is sampled at $40 \mathrm{~ms}$ intervals during a complete heart cycle. The cross section is digitised and stored in the computer memory. The step motor is then activated and rotates the control knob of the oesophageal multiplane probe or the precordial transducer $2^{\circ}$ to the next cross section where the same logic is followed. To encompass the whole heart (or region), 90 sequential standardised cross sections must be obtained each during a complete heart cycle.

\section{The image processing}

After acquisition, cross sectional images are resampled in the correct sequence according to their ECG phase in volumetric data sets $(256 \star 256 \star 256 \star$ pixel/each 8 bit $)$. The image data set acquired by rotational scanning must be resampled for conversion from a polar to a Cartesian coordinate system (fig 1). Mathematical interpolation is used to create pixel intensities to generate the three dimensional image elements to fill the gaps. Finally, methods for removing artefacts and improving the contrast are necessary. Several algorithms are applied to reduce noise, enhance edges, and reduce spatial artefacts, and this step is important for the overall quality of the final three dimensional reconstruction.

\section{The display of three dimensional reconstruction}

Different displays from three dimensional datasets can be produced: (1) a two dimensional display from individual selected cut planes (anyplane echocardiography); or from parallel short axis cuts (along a defined long axis); or from long axis cuts (up to eight different views, at different angle increments); (2) a volume rendered technique: from any defined cut plane, different algorithms are applied to represent the information in space.

For volume rendered display, a threshold value is used to identify the borders of the object of interest and to separate cardiac structures from the blood pool and background. Brightness and shading are used to give the perception of depth, and different algorithms can be combined to obtain the best results according to the region of interest. ${ }^{12}$ The final display of the images has a close resemblance to the anatomy, and relationships among the different structures are readily appreciated. Details of a valve leaflet can be discerned and dynamic three dimensional visualisation enhances the perception of the normal and affected structure.

\section{The examination procedure}

The rotational approach allows the acquisition of the images with the same multiplane probe used for the standard examination, thus allowing the procedure to be more easily tolerated by the patient than other acquisition techniques. ${ }^{11}$ Precautions, preparation of the patients, and insertion of a multiplane probe have been described, ${ }^{1}$ and acquisition of the cross sectional images for three dimensional reconstruction is part of the standard diagnostic study. The settings of the ECG and respiration gating are selected after two or three rotational scans to assure that the scanned 
Table 2 Advantages of three dimensional echocardiography

(1) Standardised examination procedure

(2) Objective imaging

(3) Improved accuracy of qualitative information

(4) New quantitative indices of cardiac function

(5) Preoperative simulation of repair

volume encompasses the structures of interest. The rotation of the scan plane is switched to the step motor under control of the steering logic to sample the cross sectional images.

Transthoracic studies are performed with the transducer system in the parasternal or apical position. After the standard examination, the transducer is mounted in the cylindrical holder connected to the computer algorithm. The operator has to find the centre axis around which the imaging plane is rotated to include the structure of interest in the centre of the sector during the whole rotation. Since the spatial coordinate system changes with transducer movement, the operator must be able to keep the transducer stationary during the procedure. Furthermore, movement of the patient during the image acquisition must be prevented by thoroughly explaining the procedure before the study.

Positioning the probes, the calibration process, and recording and storage of the cross sectional images requires approximately 7-10 minutes more than the time needed for a standard cross sectional examination.

Three dimensional reconstruction is performed off line and processing times vary between 30 and 45 minutes, depending how difficult it is to select the optimal cut planes to visualise a given structure or region of interest, as there may be significant anatomical variability between patients and countless cut planes can be selected. Guidelines to identify approximate cutting planes in various categories of cardiac disorder have recently been proposed. ${ }^{13}$ Valve leaflets can be observed from above or from below and the septa from the right or left side, similar to the surgical views.

\section{Current experience and advantages of} three dimensional echocardiography The initial results of the application of three dimensional echocardiography in humans are promising. ${ }^{81014.19}$ Advantages of three dimensional echocardiography are reported in table 2 .

\section{“ANYPLANE" CROSS SECTIONAL IMAGING}

The limitations of acoustic access and registration of individual cross sectional images can potentially be overcome by three dimensional echocardiography. From the original three dimensional dataset, new individually optimised and otherwise unobtainable image planes can be computed and displayed in motion (dynamic anyplane echocardiography). A zoom facility allows visualisation of detailed structures. Slicing of a given structure can be performed with parallel scanning in a way similar to computed tomography or magnetic resonance imaging. Up to eight longitudinally cut planes with different angle interval can be simultaneously displayed for better spatial appreciation (fig 2). Furthermore, the final assessment of cavity dimensions or the evaluation of a given structure will be more objective and less operator dependent.

\section{QUANTIFICATION}

Volume measurements

Various cross sectional approaches for measuring left ventricular volume have been proposed, but all make some assumptions about
Figure 2 Computer generated long axis views. From the three dimensional dataset, a short axis reference cut is selected and eight cut lines at $22 \cdot 5^{\circ}$ intervals are represented (panel $A$ ). The corresponding long axis cuts are displayed in panels cuts - .
Anyplane echocardiography

Left ventricular long axis views
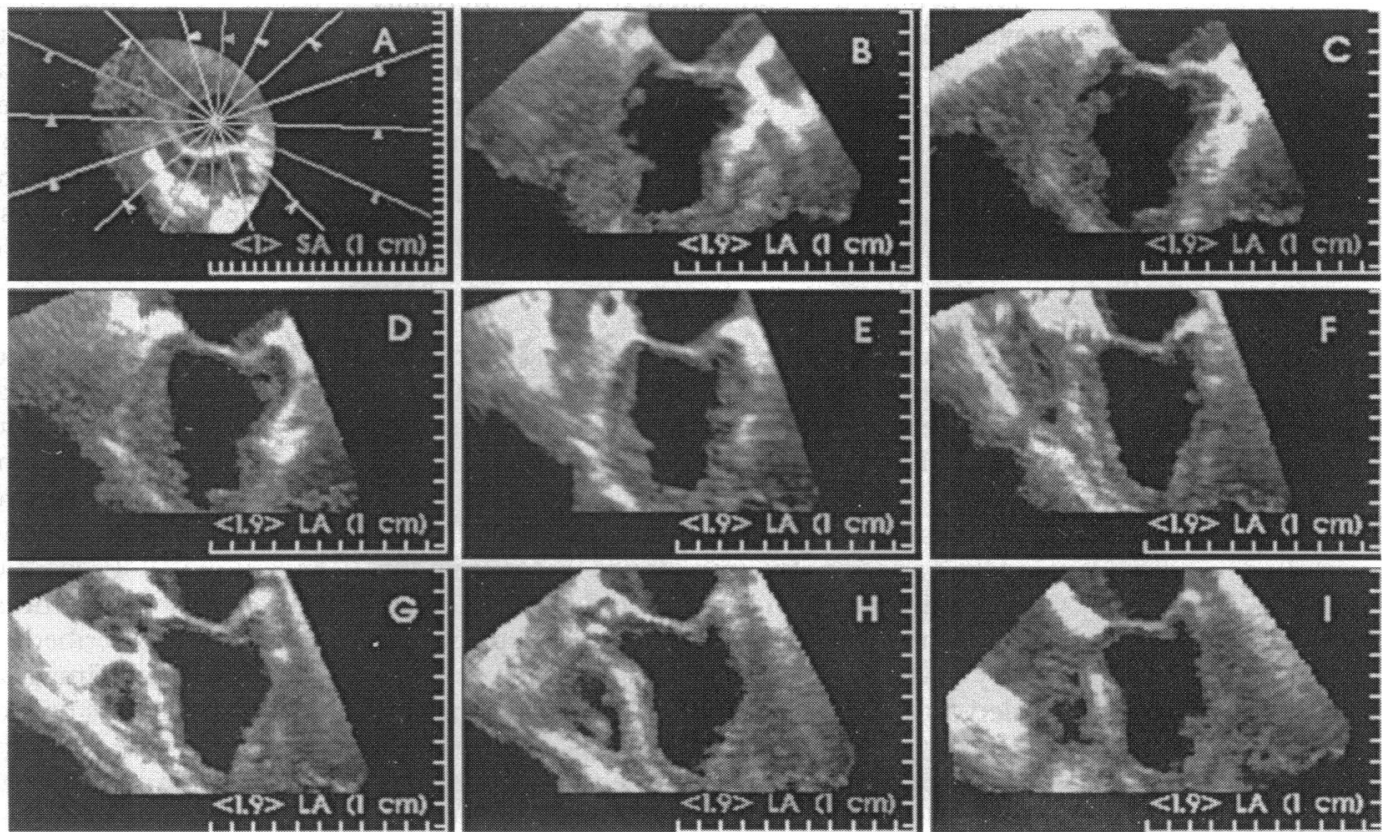
Figure 3 Volume measurement. From the

three dimensional dataset,

a long axis cut plane of the

heart is selected (left

panel). Short axis cut

planes (perpendicular to

the long axis) at

selected and the

corresponding images

displayed. Three

representative short axis

images are shown in the

middle panel. For each

endocardial contour is

drawn and the calculation

is automatically done.

Slice by slice, the system

does a summation of the

corresponding subvolumes.

Finally, all contour lines

can be displayed in their

spatial orientation and

rotated on the screen for

better representation (right panel). predetermined distance are

short axis image, the

of the volume of each slice

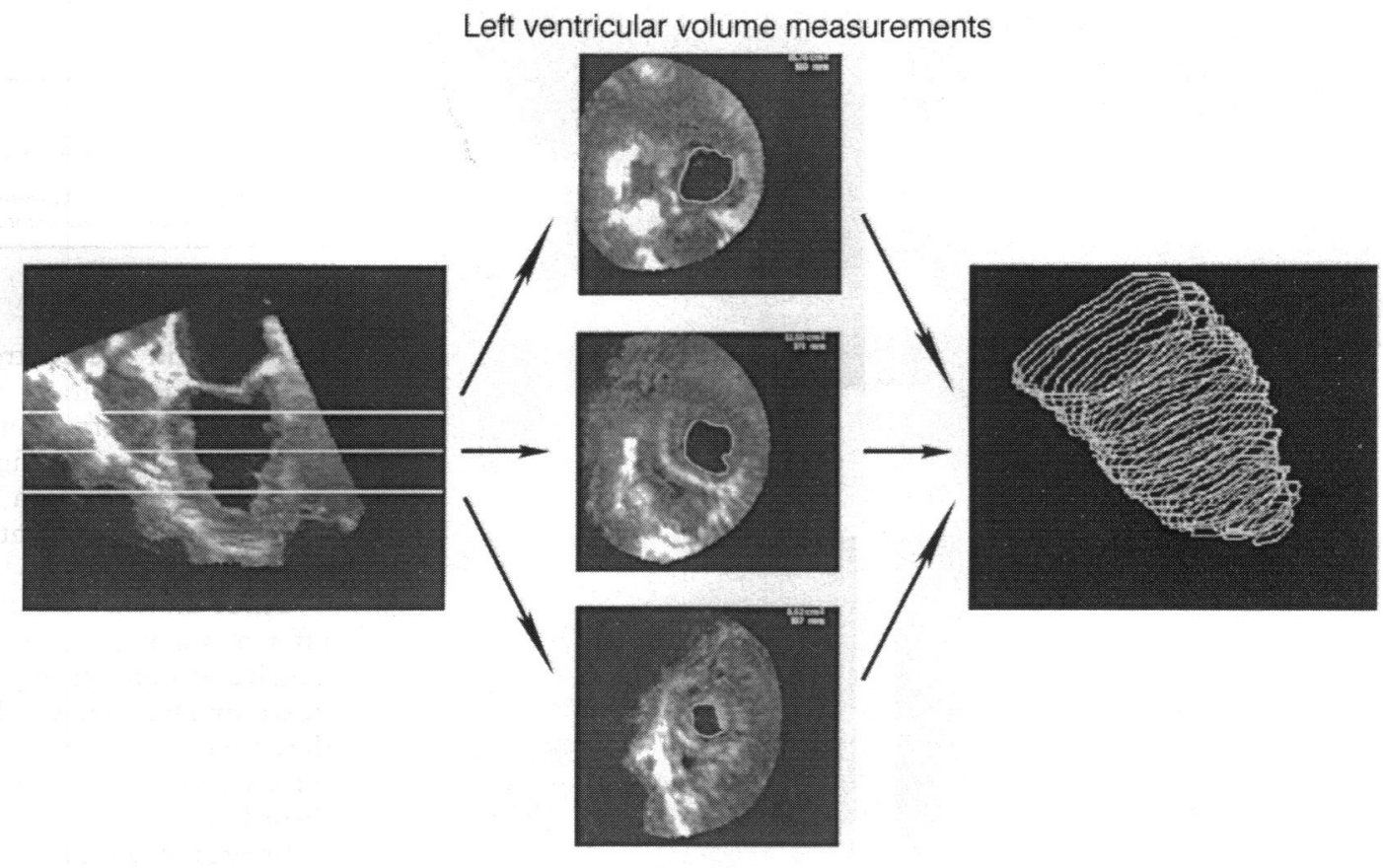

cavity shape. ${ }^{20}$ With standard cross sectional echocardiography only a limited number of planes can be obtained; thus a theoretical geometrical model must be assumed which is often far from the reality. From the three dimensional dataset, orthogonal long axis cut planes can be automatically selected, which
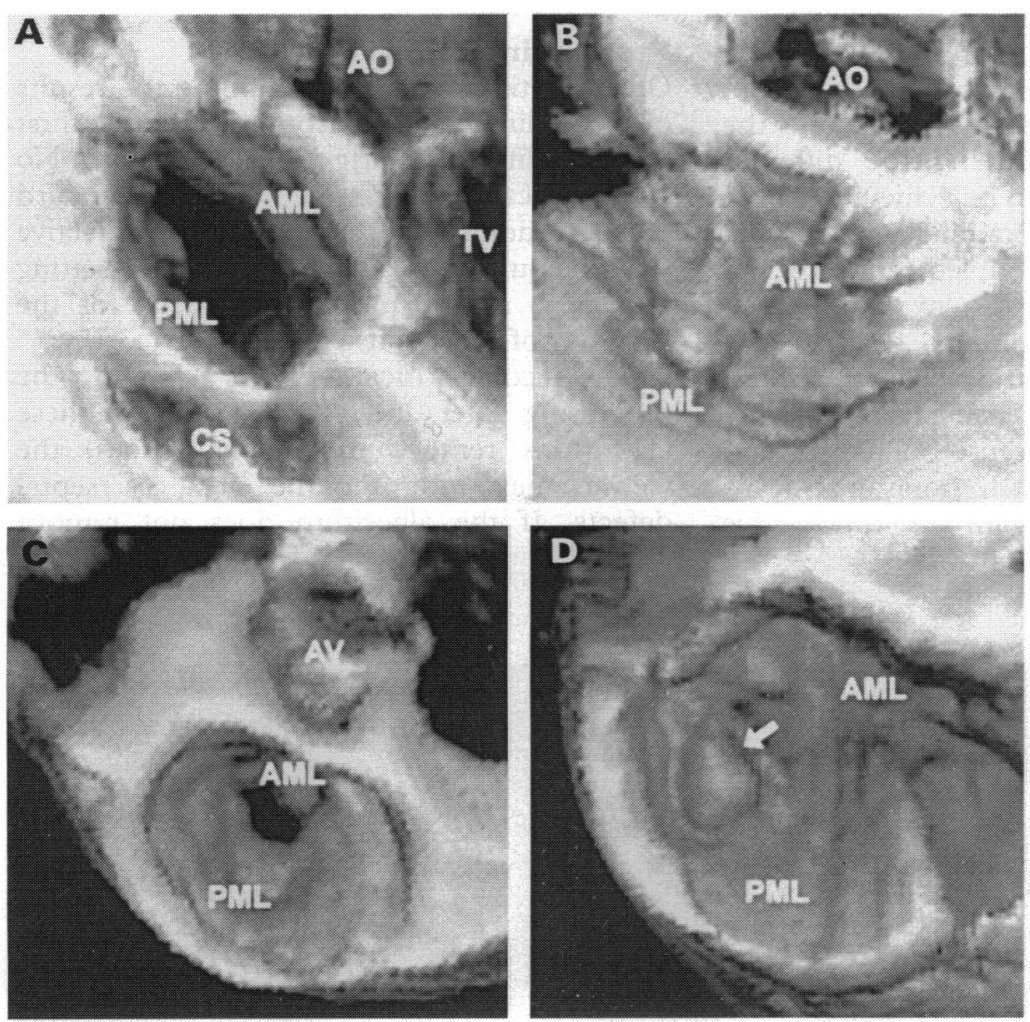

Figure 4 Three dimensional reconstruction of the mitral valve (precordial acquisition, $A$ and $B$; transoesophageal acquisition, $C$ and D). An unroofed view of the left atrium shows a normal valve in diastole (panel $A$ ) and in systole (panel B). The orifice of a stenotic mitral valve in diastole is represented in panel $C$. In panel $D$, a prolapse of the lateral scallop of the posterior leaflet is clearly seen in systole (arrow). $A O=$ aorta; $A V=$ aortic valve; $A M L=$ anterior mitral leaflet; $C S=$ coronary sinus; $P M L=$ posterior mitral leaflet; $T V=$ tricuspid valve. partially compensates the geometric assumptions involved in biplane methods.

The major advantage of three dimensional echocardiography over standard cross sectional imaging relies on more objective assessment of ventricular shape and size, since it does not depend on any specific transducer location or orientation plane. Thus three dimensional echocardiography should be able to define chamber volume in an accurate and reproducible manner. Manual tracings of endocardial borders from a series of parallel short axis cut planes of the left ventricle at variable intervals allow computation of left ventricular volumes independent of theoretical models. Volumes of individual slices are calculated $(V=A \times h$, where $A=$ area of the slice, and $\mathrm{h}=$ distance between adjacent cut planes) and summed to obtain the total volume (fig 3). Serial studies with three dimensional echocardiography will provide more insight into the natural history of complex cardiac pathology and into the rate of progression of its severity (for example, ventricular remodelling).

\section{Distance measurements}

The different surface points of a three dimensional reconstruction are not in one plane. Thus a distance measurement must always take the depth into account. From the volume rendered display, the definition of a start and end point of the distance to be measured will result in the computation of the distance in the voxel space.

\section{Area measurements}

Although area measurements can only be applied to cross sectional images, three dimensional echocardiography permits sectioning of the heart in any desired orientation. Thus cut planes can be selected which cross section the structure in the desired optimal orientation. This makes orifice area measurement more accurate. 

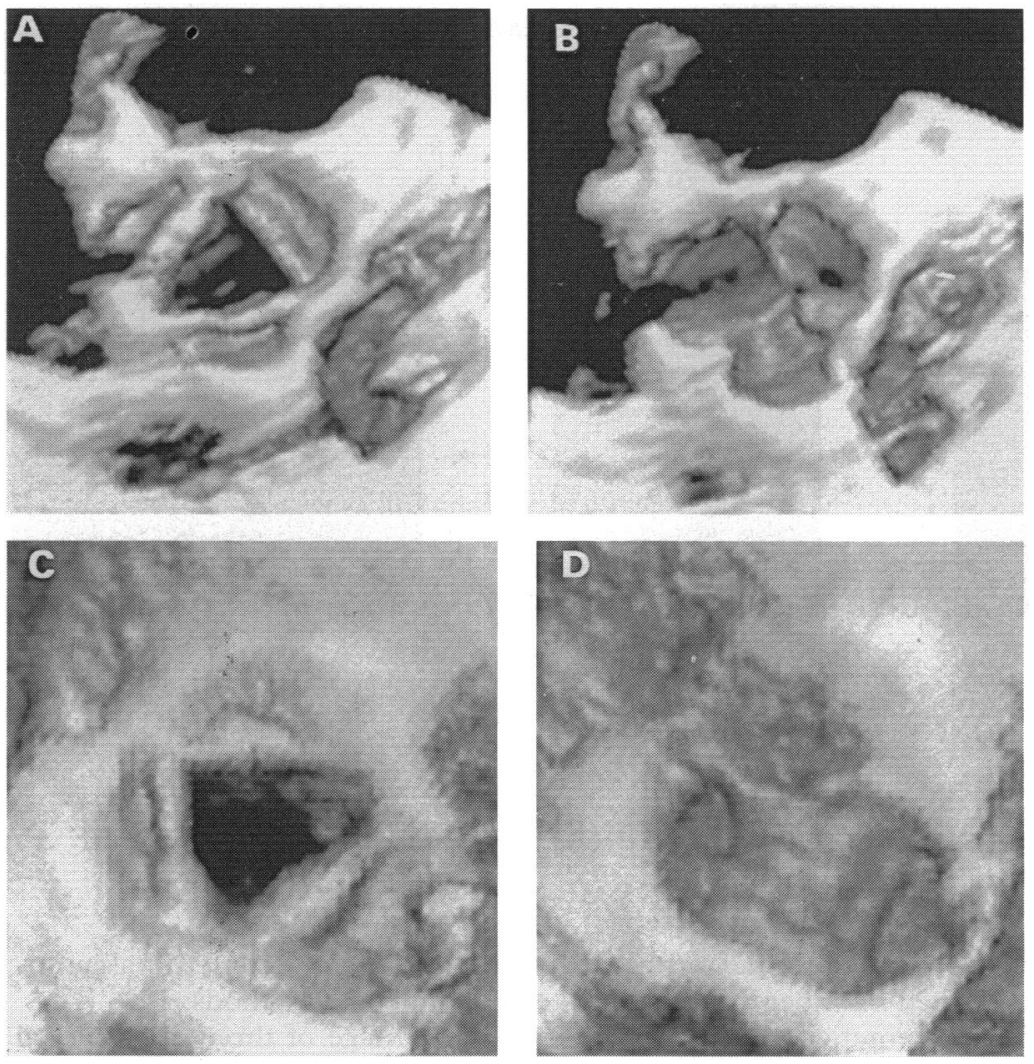

Figure 5 Normal aortic valve. Three dimensional reconstruction from transoesophageal $(A$ and $B)$ and precordial ( $C$ and $D)$ acquisition. View of the valve from above shows the open leaflets in systole (panel $A$ and $C$ ) and its typical arrangement in diastole (panel $B$ and D).
Table 3 Present problems with three dimensional echocardiography

(1) Acquisition of processing time (storage space, computing power)

Transducer stability during acquisition

3) Limited resolution

Susceptibility to background noise artefacts

(5) No on line three dimensional representation

remaining in the centre of the sector during the whole acquisition, which allows adequate sampling of information and subsequent optimal reconstruction (fig 4 ). The aortic valve can be observed from above or from below, which enhances evaluation of leaflet morphology (fig 5).

Three dimensional echocardiography would offer a major potential role for the comprehensive evaluation of patients with congenital heart disease (fig 6). Dynamic volume rendered reconstructions provide accurate spatial information, especially in complex congenital disorders. ${ }^{22}$

Images of acquired or congenital pathology can be displayed from views which simulate surgical exposure of the affected structure. Thus, in patients who are candidates for cardiac surgery, three dimensional echocardiography can provide a dynamic view of the surgical anatomy of the heart. The surgeon can observe the affected structure in a way similar to that which he will find later in the operating room. Clearly this will facilitate the surgical planning of the procedure.

MORPHOLOGICAL DIAGNOSIS

At the Thoraxcenter, our experience with an integrated system for three dimensional reconstruction shows that the potential goal of the objective display of the anatomy and the complex relationships among the different structures of the heart can be achieved. ${ }^{8 \cdot 10}$

The possibility of obtaining unrestricted cross sectional images coupled with the volume rendered dynamic display allow us to explore fully the morphological features of a given structure. The mitral valve is an ideal structure for three dimensional reconstruction. ${ }^{21}$ With both the transoesophageal and the transthoracic approach, rotational scanning can be performed with the mitral valve

\section{Present limitations (table 3)}

Of the critical factors determining the results of three dimensional reconstruction, the first is the quality of the original cross sections. No software can compensate for low standard cross sectional image quality and defective image acquisition. An appropriate gain setting and basic image quality are crucial for the accuracy of the reconstruction.

The second critical factor is related to the processing filter and segmentation. If these algorithms remove too many echoes, the viewer may interpret the gaps as septal defects. If the algorithm does not remove
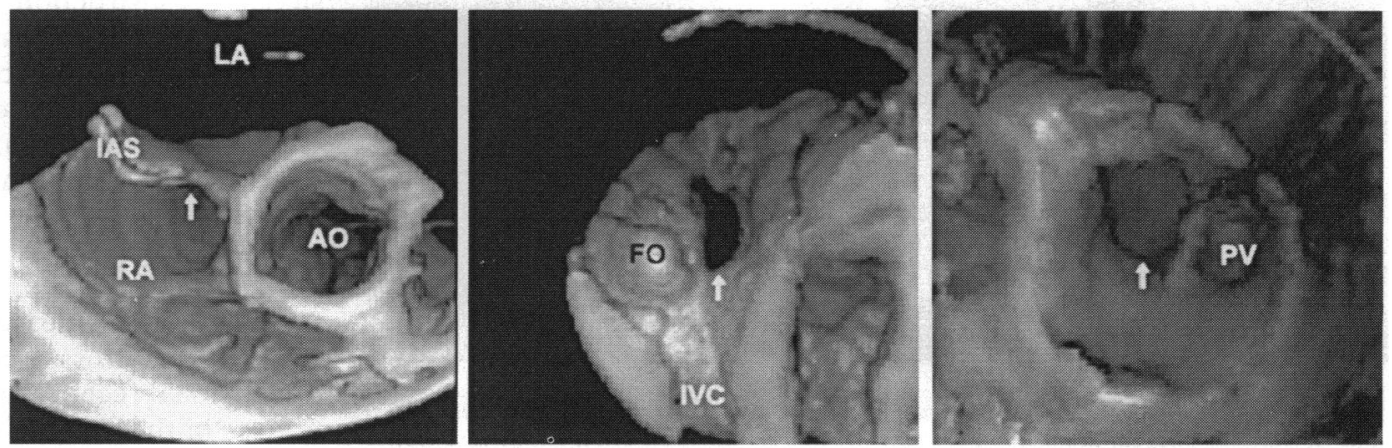

Figure 6 Three dimensional reconstruction from a patient with atrial septal defect (arrow). In the left panel, volumerendered display of a standard transoesophageal cross section is represented. Cut planes parallel to the interatrial septum show the defect as viewed from the right (middle panel) and from the left (right panel) atrium. $A O=$ aorta; FO = fossa ovalis; $I A S=$ interatrial septum; IVC = inferior vena cava; $L A=$ left atrium; $P V=$ pulmonary vein; $R A=$ right atrium. 
enough noise, confusing echoes will appear in the reconstruction and can be mistaken for vegetation or thrombus.

Thirdly, the resolution in the conical volume is non-uniform in two dimensions, worsening progressively from the central axis to the lateral field and from the top to the bottom. Thus when a tangential cut plane is computer reconstructed and displayed the resolution will be different in any given point of this cut plane and may affect the interpretation of the images.

\section{Future directions}

The development of new software will further improve the final image quality. To date, three dimensional echocardiography has not provided information on blood flow. The possibility of colour Doppler flow recording combined with the volume rendered tissue reconstruction and its dynamic three dimensional display will provide a quantitative assessment of the regurgitant flows or shunts in the future, and greatly enhance the comprehensive assessment of valvular lesions. This topic is under current investigation. ${ }^{23}$

\section{Appendix}

TERMINOLOGY

Acquisition: Technique for collecting the data.

Predetermined acquisition: acquisition is performed moving the transducer in a prescribed sequential manner.

Rotational acquisition: movement of the transducer around the axis of the ultrasound beam.

Processing: Series of steps to extract the region of interest from its surroundings.

Three dimensional dataset: The volume of the acquired and processed images.

Display: Techniques for display of the images on the output screen.

Static display: representation of the images in stop frame.

Dynamic: representation of the images in motion. Anyplane two dimensional imaging. The possibility of obtaining countless cut planes from the original three dimensional dataset.

Rendering: The way in which the three dimensional structures are presented.

Volume rendering. display of three dimensional images maintaining the information from the whole volume and thus tissue in grey scale.

1 Roelandt JRTC, Thomson IR, Vletter WB, Brommersma P, Bom N, Linker DT. Multiplane transesophageal echocardiography: latest evolution in an imaging revolution. $f$ Am Soc Echocardiogr 1992;5:361-7.

2 Dekker DL, Piziali RI, Dong E. A system for ultrasonically imaging the human heart in three dimensions. Comput Biomed Res 1974;7:544-53.

3 Gosh A, Nanda NC, Maurer G. Three-dimensional reconstruction of echocardiographic images using the rotation method. Ultrasound Med Biol 1982;6:655-61.
4 Geiser EA, Ariet M, Conetta DA, Lupkiewicz SM Christie LG, Conti CR. Dynamic three-dimensional echocardiographic reconstruction of the intact human echocardiographic reconstruction of the intact human patients. Am Heart $\mathcal{f} 1982 ; 103: 1056-65$.

5 Raichlen JS, Trivedi SS, Herman GT, St John Sutton MG, Reichek N. Dynamic three-dimensional reconstruction of the left ventricle from two-dimensional echocardiograms. I Am Coll Cardiol 1986;8:364-70.

6 Belohlavek M, Foley DA, Gerber TC, Kinter TM Greenleaf JF, Seward JB. Three- and four-dimensiona cardiovascular ultrasound imaging: a new era for echocardiography. Mayo Clin Proc 1993;68:221-40.

7 Nanda NC, Pinheiro L, Sanyal R, Rosenthal S, Kirklin JK. Multiplane transesophageal echocardiographic imaging and three-dimensional reconstruction. Echocardiography 1992;9:667-76.

8 Roelandt J, Ten Cate FJ, Bruining N, Salustri A, Vletter WB, Mumm B, et al. Transesophageal rotoplane echoCT. A novel approach to dynamic three-dimensional CT. A novel approach to dynamic three-dim

9 Roelandt JRTC, TenCate FJ, Vletter WB, Taams MA Ultrasonic dynamic three-dimensional visualization of the heart with a multiplane transesophageal imaging transducer. F Am Soc Echocardiogr 1994;7:217-29.

10 Roelandt J, Salustri A, Bekkering L, Bruining N, Vletter WB. Precordial three dimensional echocardiography with a rotational imaging probe (precordial rotoplane echo-CT). Methods and initial clinical experience. Echocardiography (in press).

11 Salustri A, Roelandt JRTC. Ultrasonic three-dimensional reconstruction of the heart. Ultrasound Med Biol (in press).

12 Drebin R, Carpenter L, Harrahan P. Volume rendering. SIGGRAPH'88 1988:65-74.

13 Pandian NG, Roelandt J, Nanda N, Sugeng L, Cao Q Azevedo J, et al. Dynamic three-dimensional echocardiography: methods and clinical potential. Echocardiography graphy: methods and

14 Rivera JM, Siu SC, Handschumacher MD, Lethor JP Guerrero JL, Vlahakes GJ, et al. Three-dimensional reconstruction of ventricular septal defects: validation studies and in vivo feasibility. F Am Coll Cardiol 1994; 23:201-8.

15 Handschumacher MD, Lethor JP, Siu SL, Mele D, Rivera $\mathrm{JM}$, Picard MH, et al. A new integrated system for threedimensional echocardiographic reconstruction: development and validation for ventricular volume with application in human subjects. $\mathcal{F}$ Am Coll Cardiol 1993; 21:743-53.

16 Pandian NG, Nanda NC, Schwartz SL, Fan P, Cao Q, Sanyal R, et al. Three-dimensional and four-dimensional transesophageal echocardiographic imaging of the heart and aorta in humans using a computed tomographic imaging probe. Echocardiography 1992;9:677-87.

17 Belohlavek M, Foley DA, Gerber TC, Greenleaf JF, Seward JB. Three-dimensional ultrasound imaging of the atrial septum: normal and pathologic anatomy. $\mathscr{f} \mathrm{Am}$ the atrial septum: normal and

18 Raqueno R, Ghosh A, Nanda NC, Schott J, Moos S. Four-dimensional reconstruction of two-dimensional echocardiographic images. Echocardiography 1989;6: 323-37.

19 Schwartz SL, Cao Q, Azevedo J, Pandian NG. Simulation of intraoperative visualization of cardiac structures and study of dynamic surgical anatomy with real-time threedimensional echocardiography. Am $\mathcal{F}$ Cardiol 1994;73. 501-7.

20 American Society of Echocardiography Committee on Standards, Subcommittee on quantitation of twodimensional echocardiograms. Recommendations for quantitation of the left ventricle by two-dimensional echocardiography. F Am Soc Echocardiogr 1989;2: 358-67.

21 Levine RA, Handschumaker MD, Sanfilippo AJ, Hagege AA, Harrigan P, Marshall JE, et al. Three-dimensional echocardiographic reconstruction of the mitral valve, with implications for the diagnosis of mitral valve prolapse Circulation 1989;80:589-98.

22 Vogel M, Lösch S. Dynamic three-dimensional echocardiography with a computed tomography imaging probe: initial clinical experience with transthoracic application in infants and children with congenital heart defects. $B$ Heart $\mathcal{F}$ 1994;71:462-7.

23 Esakof D, Cao Q, Tardif JC, Azevedo J. Dynamic threedimensional reconstruction and display of mitral and aortic regurgitant flow jets and the left heart chamber from transthoracic echocardiograms in a clinical setting. Circulation 1993;88(Part 2):I-349. 